\section{Novel mutations in NOTCH2 gene in infants with neonatal cholestasis}

Eliana Shaul,1

Debora Kogan-Liberman, ${ }^{2}$

Stephanie Schuckalo, ${ }^{3}$ Dominique Jan, ${ }^{4}$

Michelle Ewart, 5 Trang Nguyen, ${ }^{2}$

Mercedes Martinez, 6

Nadia Ovchinsky 2,3

1Department of Pediatrics, Children's

Hospital at Montefiore, Bronx, NY;

2Division of Pediatric Gastroenterology,

Hepatology and Nutrition, Children's

Hospital at Montefiore, Bronx, NY;

3Division of Pediatric Gastroenterology,

Hepatology, and Nutrition, Goryeb

Children's Hospital - Atlantic Health

System, Morristown, NJ; ${ }^{4}$ Department

of Pediatric Surgery, Children's

Hospital at Montefiore, Bronx, NY;

5Division of Surgical Pathology,

Montefiore Medical Center, Bronx, NY;

6Division of Pediatric Gastroenterology,

Hepatology, and Nutrition, Morgan

Stanley Children's Hospital of New

York, NY, USA

\begin{abstract}
One cause of neonatal cholestasis (NC) is paucity of intrahepatic bile ducts which can be associated with Alagille syndrome or non- syndromic. Alagille syndrome is caused by autosomal dominant mutations in the Notch signaling pathway ligand Jagged 1 in $94 \%$ of patients and mutations in the NOTCH 2 receptor in $<1 \%$ of patients. This is a retrospective case series studying infants with neonatal cholestasis found to have variants of unknown significance (VOUS) in NOTCH2. Sorting intolerant from tolerant (SIFT) and polymorphism phenotyping (PolyPhen) were utilized to predict a damaging effect. Five infants with NC without other features of Alagille syndrome were found to have one copy of a VOUS in $\mathrm{NOTCH} 2$, predicted to be damaging by SIFT and PolyPhen. Our cases support the notion that NOTCH2 mutations may result in hypoplastic biliary system. Further characterization of these variants is important to assist with our clinical approach to NC.
\end{abstract}

\section{Introduction}

Impaired flow and excretion of bile in the newborn period results in accumulation of biliary components and leads to neonatal cholestasis (NC). ${ }^{1}$ Paucity of intrahepatic bile ducts (PIBD) is one cause of NC and can be part of a an autosomal dominant familial cholestatic syndrome called Alagille Syndrome (ALGS) ${ }^{2}$ or can be nonsyndromic. ${ }^{3}$ In $94 \%$ of patients with ALGS, mutations in the gene for the Notch signaling pathway ligand Jagged1 (JAGl) are found. Furthermore, mutations in $\mathrm{NOTCH} 2$, a receptor in the Notch signaling have been found in patients with ALGS. ${ }^{4}$ We report 5 patients, including 2 siblings (patients $1 \& 2$ ), with $\mathrm{NC}$ without any other features of ALGS, who were found to have novel variants of unknown significance (VOUS) in the NOTCH2 gene.

\section{Methods}

This is a retrospective case series of 5 patients with NC. Data was obtained through a chart review, including electronic medical records, imaging and pathology specimens. Genetic testing for known pediatric cholestatic disorders was done via Emory Genetics Laboratory (EGL) Genetic Cholestasis Panel. Direct sequencing of the amplified captured regions was performed using next generation short base pair read sequencing (NGS). Exons with inadequate quality or coverage by NGS were assessed with Sanger sequencing. We utilized sorting intolerant from tolerant (SIFT) and polymorphism phenotyping (PolyPhen), which are silico bioinformatic tools that predict the likelihood of pathogenicity of missense variants. ${ }^{5}$ SIFT predicts whether an amino acid substitution is likely to affect protein function based on sequence homology and similarity between the alternate amino acids and then predicts if the amino acid change is either 'tolerated' or 'deleterious'. PolyPhen-2 predicts the effect of an amino acid substitution on the structure and function of a protein using sequence homology, 3D structures where available, and a number of other databases and tools and then provides a qualitative prediction of 'probably damaging', 'possibly damaging', 'benign' or 'unknown'. This study was deemed exempt by The Albert Einstein College of Medicine Institutional Review Board.

\section{Case Report}

Five male infants with $\mathrm{NC}$ between the age of 2 and 8 weeks were found to have one copy of VOUS in NOTCH 2 gene (Table 1). None of the infants had known risk factors for cholestasis. They all present-
Correspondence: Nadia Ovchinsky, Division of Pediatric Gastroenterology, Pediatric Hepatology, Children's Hospital at Montefiore, 3411 Wayne Ave, 7th Floor, Bronx, NY 10467, USA.

Tel.: +1.718-741-2332 - Fax 718-515-5426.

E-mail: novchins@montefiore.org

Key words: Paucity of intrahepatic bile ducts; NOTCH2; Neonatal Cholestasis.

Contributions: ES: study concept and design, drafting of the manuscript, analysis and interpretation of results, literature review. DKL: drafting the work and revising it critically for important intellectual content. SS: acquisition of data and chart review, technical support. DJ: Acquisition of data and surgical findings. ME: Acquisition of data and pathological findings. TN: Acquisition of data and chart review, technical and administrative support. MM, critical revision of the manuscript for important intellectual content. NO: study concept and design, critical revision of the manuscript for important intellectual content, final approval of the version published, agreement to be accountable for all aspects of the work in ensuring that questions related to the accuracy or integrity of any part of the work are appropriately investigated and resolved.

Conflict of interest the authors declare no potential conflict of interest.

Funding: none.

Received for publication: 14 June 2019.

Revision received: 22 June 2019

Accepted for publication: 29 July 2019.

This work is licensed under a Creative Commons Attribution NonCommercial 4.0 License (CC BY-NC 4.0)

(C) Copyright: the Author(s), 2019

Licensee PAGEPress, Italy

Pediatric Reports 2019; 11:8206

doi:10.4081/pr.2019.8206

ed with jaundice, acholic stools and without growth failure. Patients 1 and 2 were siblings with identical mutations. Liver biopsy was done in 3 patients demonstrating paucity of bile ducts in 2 patients (Figure 1) and mild ductular proliferation in the third. In all patients SIFT predicted the mutations to be deleterious and PolyPhen predicted them to be probably damaging. Further workup for other features of ALGS, including an echocardiogram, eye exam and X-Ray of the spine, were all within normal limits. Additionally, workup for common etiologies of NC including infectious, anatomic, metabolic, and genetic etiologies was unremarkable. All patients demonstrated 
improvement in liver disease over time and remain asymptomatic.

\section{Discussion}

$\mathrm{NC}$ is caused by diminished flow and excretion of bile. ${ }^{1}$ Symptoms typically include jaundice, dark urine, acholic stools and hepatomegaly. Cholestasis can occur due to infectious, genetic, anatomic and metabolic causes, generally caused by impairment of hepatobiliary transport, intermediary metabolism, storage disorders or bile duct dysgenesis. ${ }^{6}$ PIBD is one cause of $\mathrm{NC}$ and is defined by a specimen from a liver biopsy shows a loss of intrahepatic bile ducts in more than $50 \%$ of portal tracts in a specimen that contains at least 10 portal tracts. ${ }^{7}$ PIBD can be part of a genetic syndrome, ALGS or can caused other rare metabolic diseases, infections such as CMV, or can be idiopathic non-syndromic paucity.2,3 A liver biopsy in patients with ALGS typically demonstrates paucity of the intrahepatic bile ducts, however in newborns with ALGS, bile duct paucity is not always present and instead ductal proliferation can be found. ${ }^{8}$ The diagnosis of ALGS requires the presence of bile duct paucity with three of five major clinical features including liver disease, vertebral abnormalities, congenital heart defects, ocular anomalies and characteristic facial features. ${ }^{9}$ ALGS is an autosomal dominant inherited disorder with highly variable expressivity, therefore the disease penetration and severity of the affected organs can vary significantly. ${ }^{10}$

Notch pathway interactions are critical for determination of cell fates and differentiation in early development. The Notch system includes of four transmembrane Notch receptors (Notch 1, 2, 3, 4) and two types of ligands Jagged (Jag 1,2) or Deltalike (Dll 1, 3, 4). The Notch pathway is involved in several stages of bile duct morphogenesis including in the expression of cholangiocytes-specific markers committing cells to the biliary lineage. ${ }^{11}$

In $94 \%$ with ALGS, mutations in $J A G 1$ are found, 4 while mutations in NOTCH2 have been described in in a small number of patients who met diagnostic criteria for ALGS without $J A G 1$ mutations. ${ }^{9,12}$ In one study, Kamath et al describe a cohort of $J A G 1$-negative individuals with clinical features suggestive of ALGS screened for $\mathrm{NOTCH} 2$ mutations. Eight patients with a NOTCH2 mutations were identified and only three met classic criteria for ALGS. Of the remaining five individuals, four had two typical ALGS diagnostic features and one
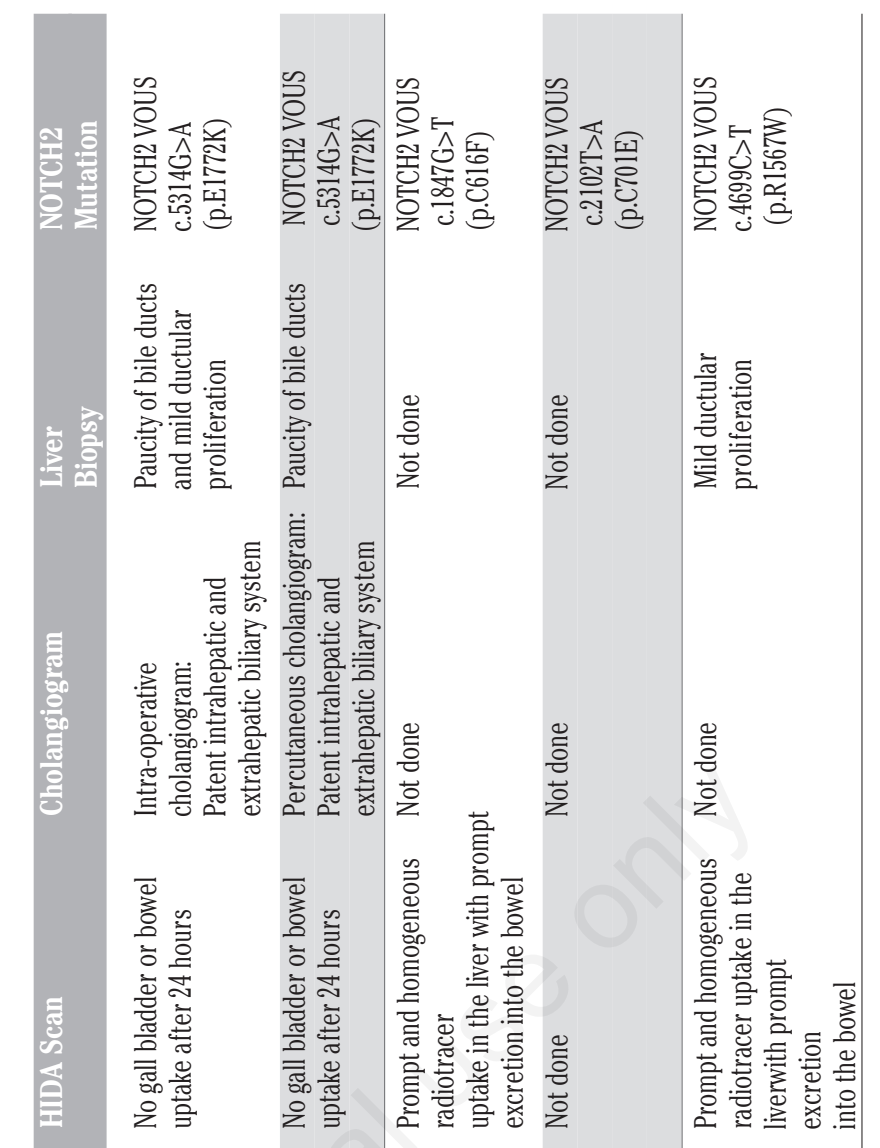

(n)

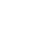



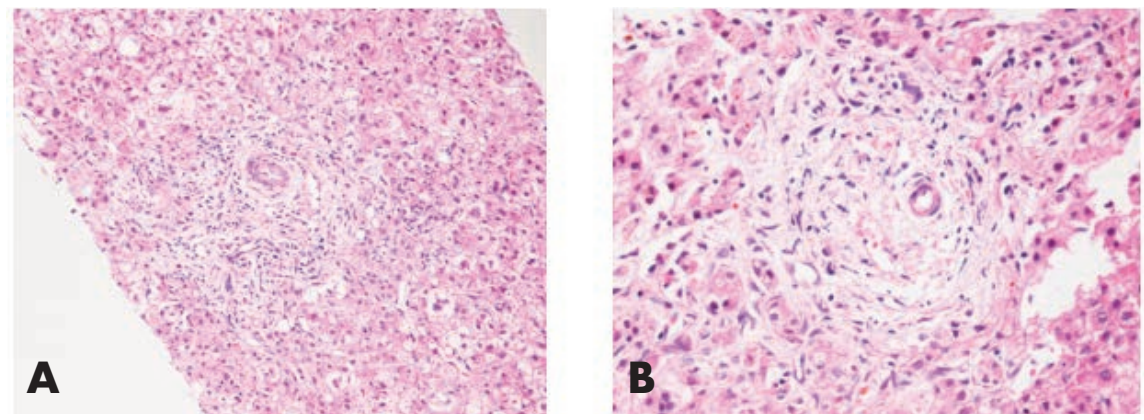

Figure 1. Histological findings demonstrating PIBD in patient 1. A) Portal area with mid chronic inflammation with visible hepatic artery branch and portal vein branches without any native bile ducts. There are surrounding giant cell transformation of hepatocytes. B) Another portal area showing hepatic artery and portal vein without a native bile duct.

patient had bile duct paucity with no other syndromic features. ${ }^{13}$ This study found that in their patients the variety of clinical features associated with $\mathrm{NOTCH} 2$ mutations differed from $J A G 1$ mutations, with a lower prevalence of butterfly vertebrae and facial features.

\section{Conclusions}

These five patients, along with one patient described by Kamath et al., suggest that $\mathrm{NOTCH} 2$ mutations may be related to isolated NC or PIBD without other features of ALGS. Since the Notch pathway is involved in bile duct morphogenesis, these cases stipulate that NOTCH2 mutations may result in hypoplastic biliary system and intrahepatic bile duct paucity. Furthermore, this series suggests that $\mathrm{NOTCH} 2$ mutations may provide the genetic basic to explain the clinical finding in infants with isolated neonatal cholestasis without other features of ALGS. It is also crucial to consider that the highly variable expression and reduced penetrance seen with ALGS could be influencing clinical presentation seen in our patients. Screening for NOTCH2 muta- tions in patients with NC is warranted and in those with PIBD even if they only fulfil partial criteria for ALGS. Validation of these finding in a larger human cohort and further characterization of the NOTCH2 variants in an animal model is especially important to understand the clinical application of these results. Further investigation will need to be done in order to determine whether these $\mathrm{NOTCH} 2$ variants are truly deleterious as the current data presented are insufficient to determine causality of these variants in neonatal cholestasis. Increasing availability of genetic testing and ability to link the clinical finding with previously unrecognized mutations provide a platform for more precise and less invasive approach to achieve a diagnosis in this vulnerable population.

\section{References}

1. Feldman AG, Sokol RJ. Neonatal cholestasis. Neoreviews 2013;14.

2. Zahmatkeshan M, Geramizadeh B, Haghighat M, Enteshari, H. Paucity of Intrahepatic Bile Ducts in Neonates: the First Case Series from Iran. Iran J
Pediatrics 2013;23:65-70.

3. Kenny AP, Crimmins NA, Mackay DJ, et al. Concurrent course of transient neonatal diabetes with cholestasis and paucity of interlobular bile ducts: A case report. Pediatr Dev Pathol 2009;12:41720.

4. McDaniell R, Warthen DM, SanchezLara PA, et al. NOTCH2 mutations cause Alagille syndrome, a heterogeneous disorder of the notch signaling pathway. Am J Hum Genet 2006;79:169-73.

5. Flanagan SE, Patch AM, Ellard S. Using SIFT and PolyPhen to predict loss-of-function and gain-of-function mutations. Genet Test Mol Biomarkers 2010;14:533-77.

6. Suchy FJ. Neonatal cholestasis. Pediatr Rev 2004;25:388-96.

7. Suchy FJ. Clinical problems with developmental anomalies of the biliary tract. Semin Gastrointest Dis 2003;14:15664.

8. Saleh M, Kamath BM, Chitayat D. Alagille syndrome: clinical perspectives. Appl Clin Genet 2016;9:75-82.

9. Piccoli DA, Spinner NB. Alagille syndrome and the Jagged1 gene. Semin Liver Dis 2001;21:525-34.

10. Kamath BM, Bason L, Piccoli DA, et al. Consequences of JAG1 mutations. J Med Genet 2003;40:891-5.

11. Morell CM, Strazzabosco M. Notch signaling and new therapeutic options in liver disease. J Hepatol 2014;60:88590.

12. Guegan K, Stals K, Day M, et al. JAG1 mutations are found in approximately one third of patients presenting with only one or two clinical features of Alagille syndrome. Clin Genet 2012;82:33-40.

13. Kamath BM, Bauer RC, Loomes KM, et al. NOTCH2 mutations in Alagille syndrome. J Med Genet 2012;49:138- 